\title{
Co-operative roles for DNA supercoiling and nucleoid-associated proteins in the regulation of bacterial transcription
}

\author{
Charles J. Dorman ${ }^{1}$ \\ Department of Microbiology, Moyne Institute of Preventive Medicine, Trinity College, Dublin 2, Ireland
}

\begin{abstract}
DNA supercoiling and NAPs (nucleoid-associated proteins) contribute to the regulation of transcription of many bacterial genes. The horizontally acquired SPI (Salmonella pathogenicity island) genes respond positively to DNA relaxation, they are activated and repressed by the Fis (factor for inversion stimulation) and H-NS (histone-like nucleoid-structuring) NAPs respectively, and are positively controlled by the 0mpR global regulatory protein. The ompR gene is autoregulated and responds positively to DNA relaxation. Binding of the Fis and OmpR proteins to their targets in DNA is differentially sensitive to its topological state, whereas H-NS binds regardless of the topological state of the DNA. These data illustrate the overlapping and complex nature of NAP and DNA topological contributions to transcription control in bacteria.
\end{abstract}

\section{Nucleoid organization and gene expression in bacteria}

The genetic material in prokaryotes is located not in a nucleus, but in a cytosolic complex known as the nucleoid that is not bounded by a membrane. The structure of the bacterial nucleoid has been under investigation for decades and recently some important advances in our understanding have been reported. Several factors work to pack the chromosome into the volume occupied by the nucleoid in the cytoplasm. Among these are molecular crowding [1], negative supercoiling of the DNA [2], interaction of the DNA with NAPs (nucleoid-associated proteins) [3-6], the presence of approximately 400 looped domains within the chromosome [7] and the influence of six macrodomains that impose a superstructure $[8,9]$. The structure of the nucleoid in turn may impose compartmentalization upon the cell, restricting the diffusion of macromolecules such as mRNA to the vicinity of the genes that encode them [10]. All of these structural influences are thought to constrain gene order along the chromosome, and possibly to influence the spatiotemporal order of gene expression [11]. The realization that the bacterial nucleoid may be a highly ordered entity contrasts with earlier models in which it was regarded as being somewhat disordered, or at least poorly organized [12]. In the light of its ordered nature, how it absorbs new genes that the bacterium acquires by horizontal gene transfer is an important question. Where do these genes go within the genome, and how is their expression integrated with that of the pre-existing set of genes? We have examined

Key words: bacterial transcription, DNA gyrase, DNA supercoiling, nucleoid-associated protein transcription.

Abbreviations used: Fis, factor for inversion stimulation; H-NS, histone-like nucleoid-structuring NAP, nucleoid-associated protein; SPI, Solmonella pathogenicity island; TTSS, Type III secretion system.

1email cjdorman@tcd.ie the control of expression of the SPI (Salmonella pathogenicity island)-1 and SPI-2 virulence genes in the Gram-negative bacterium Salmonella enterica serovar Typhimurium. These genes differ from those of the core genome in having a higher $\mathrm{A}+\mathrm{T}$ content in their DNA, leading to the suggestion that they originated outside the enteric group [13]. Their regulation has been studied in detail [14], making them ideal subjects for our investigation into regulatory integration.

\section{The SPI virulence genes of Salmonella enterica}

Salmonella Typhimurium is a facultative intracellular pathogen that is adapted to the mouse, where it causes an invasive disease with characteristics similar to typhoid in humans. For this reason, Salmonella Typhimurium infection of mice has been studied for decades as a model for human typhoid. The host ingests the bacterium through the consumption of contaminated food or water and, following passage through the stomach, Salmonella Typhimurium invades the epithelial cells lining the small intestine [15]. Invasion is accomplished by means of a TTSS (Type III secretion system) whose component proteins and effector proteins are encoded by the genes of SPI-1 [16]. These genes are subject to complex regulation involving several regulatory proteins and numerous environmental signals (Figure 1). Although many of the important regulatory genes are located within SPI-1, not all are [14]. The involvement of SPI-1 in the control of genes located in the core genome is indicative of the regulatory integration of this horizontally acquired genetic element.

Salmonella Typhimurium survives in a vacuole within the invaded cells of the host. It can also survive in vacuoles within macrophages. Intracellular survival depends on a second TTSS and a second set of effector proteins encoded by SPI-2. Once again, the genes in SPI-2 are subject to 
Figure 1 | The 0mpR protein regulates virulence gene expression in Salmonello

The EnvZ histidine protein kinase is associated with the cytoplasmic membrane of the Gram-negative bacterium from where it can transmit environmental signals to its OmpR partner via protein phosphorylation. The OmpR protein in turn binds to target sequences in both the core genome (e.g. the promoter of the ompR-envZ operon) and the horizontally acquired part of the genome, such as SPI-1 and SPI-2. OmpR binds to the promoters of the hilC and hilD regulatory genes, but affects them differently. OmpR is an activator of hilC and a repressor of hilD; the latter is a gene that regulates transcription positively within both SPI-1 and SPI-2 [16,40]. The OmpR protein is a co-activator of transcription of the SPI-2 regulatory gene SSrA, and the product of this gene facilitates the activation of transcription throughout SPI-2 via its DNA-binding partner protein, SsrB $[19,20]$. These complex regulatory interactions allow the bacterium to manage SPI gene expression as the organism moves from niche to niche within the host during infection [15-18]. The four promoters studied by chromatin immunoprecipitation (see Figure 2) are highlighted by green boxes. Genes from the core genome are represented by blue arrowheads and those from the horizontally acquired part of the genome are represented by red arrowheads.

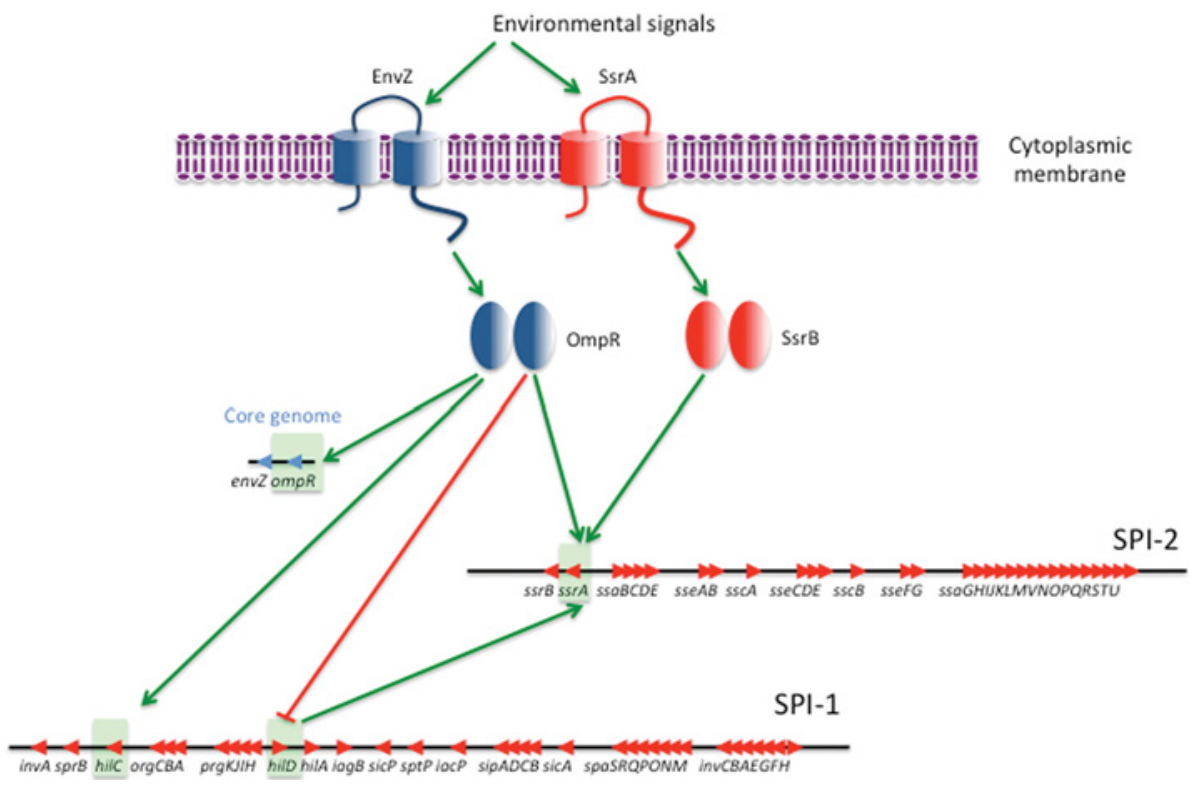

transcriptional control by a combination of SPI-2-encoded regulatory proteins and regulators that are encoded by the core genome [17] (Figure 1). There is also an element of cross-talk between the SPI-1 and SPI- 2 genes, as might be expected given that their expression is required under distinct circumstances: SPI-1 is expressed in the intestinal lumen to promote invasion, whereas SPI-2 is expressed in the vacuole to promote intracellular survival.

The regulatory proteins expressed by SPI-1 are mainly AraC-like transcription factors. Unlike the L-arabinosebinding AraC protein, these SPI-1 proteins are not known to bind ligands that impart environmental information so how their gene regulatory activity is modulated is not understood. The master regulator of SPI-2 gene transcription consists of a two-component regulatory partnership between the histidine protein kinase SsrA and the DNA-binding protein SsrB. The EnvZ/OmpR two-component regulatory system, encoded by genes in the core genome, contributes to the expression of genes in both SPI-1 and SPI-2 [18-20] (Figure 1). In addition, the NAPs Fis (factor for inversion stimulation), $\mathrm{H}-\mathrm{NS}$ (histone-like nucleoid-structuring), HU (histone-like Escherichia coli strain U93) and IHF (integration host factor) also influence SPI-1 and SPI-2 gene expression, as does the superhelical density of the DNA $[18,21,22]$.

\section{DNA supercoiling and the control of bacterial transcription}

It has been known for more than 20 years that variable DNA supercoiling influences the transcription of bacterial virulence gene expression [23]. The topology of bacterial DNA influences the structure of the nucleoid by providing a mechanism for DNA compaction through negative supercoiling. Negative supercoils are introduced by the ATP-dependent type II topoisomerase DNA gyrase. The DNA doublestrand breakage, strand passage and reannealing activities of gyrase reduce the linking number of the DNA and produce two topological effects that can influence transcription. The first concerns the creation of DNA writhing, as the axis of the DNA double helix winds around itself seeking a minimal energy conformation to counteract the torsional stress produced by DNA underwinding. Writhing has the effect of promoting long-range interactions in DNA that 
can, for example, facilitate physical contact between RNA polymerase and regulatory proteins bound elsewhere along the DNA molecule. The second topological influence that follows underwinding of the DNA is a change in the twisting of DNA around its helical axis that can facilitate the formation of single-stranded 'bubbles' as hydrogen bonds between pairs of bases are broken. This enhanced tendency on the part of the DNA to become single-stranded can facilitate the isomerization of closed transcription complexes to open ones $[24,25]$. The superhelical density $(\sigma)$ of bacterial DNA reflects the dependency of gyrase activity on the ratio of the cellular concentrations of ATP to ADP [26-28]. Superhelical density is not fixed, but varies between limits (estimated as $\sigma=-0.068$ and -0.043 ) that depend on the growth stage of the cell, its growth rate and its environmental circumstances, all of which influence the ATP/ADP concentration ratio, and hence the activity of DNA gyrase [26-28]. The promoters of the SPI genes in Salmonella Typhimurium are sensitive to changes in DNA supercoiling, and this topological sensitivity is an important component of their regulatory regime. However, its contribution must be assessed in the context of inputs from the conventional transcription factors mentioned above and those of the NAPs.

\section{NAPs and SPI gene regulation}

The promoters of the SPI genes and operons are transcriptionally repressed (or 'silenced') by H-NS. This abundant dimeric DNA-binding protein binds to structures in DNA that are specified by $\mathrm{A}+\mathrm{T}$-rich sequences, a feature of horizontally acquired genes in the enteric group [29-31]. Following initial binding, the $\mathrm{H}-\mathrm{NS}$ protein can oligomerize along individual segments of DNA or it can bridge separate segments. The formation of oligomers, whether bridged or not, is critical for H-NS to repress transcription. An impressive array of mechanisms has been described by which $\mathrm{H}-\mathrm{NS}$-mediated transcription silencing is relieved, including mechanisms in which H-NS-DNA repression complexes are disrupted by remodelling carried out by other DNA-binding proteins [32]. The Fis protein is another abundant NAP with a preference for A + T-rich DNA sequences. Unlike H-NS, which is present at all stages of growth, the Fis protein is expressed mainly in the early stages of exponential growth in well-aerated cultures of Salmonella Typhimurium [33]. Fis can act as an antagonist of the transcription silencing activity of $\mathrm{H}-\mathrm{NS}$ and is known to stimulate the transcription of the SPI genes [34].

\section{Comparison of Salmonella Typhimurium with Escherichio coli}

The Gram-negative bacteria Salmonella Typhimurium and E. coli are thought to have separated from their last common ancestor 100 million years ago [13], yet they still share genomes of comparable size with very similar gene content and gene order. In addition, the two organisms possess a very similar gene regulatory apparatus. A key difference between these bacteria lies in the possession by Salmonella Typhimurium of its pathogenicity islands, of which SPI-1 and SPI-2 are the most intensively studied. Although the two bacteria have the same NAPs, they have been found to differ in the superhelical densities of their DNA, with $\sigma$ values of -0.59 (Salmonella Typhimurium) and -0.69 (E. coli) in cultures at similar stages of growth and under identical growth conditions [35]. What are the implications of this difference in DNA supercoiling for gene expression in the two organisms?

\section{Fis and DNA supercoiling modulate SPI gene expression}

The Fis protein is encoded by the $y b d G-f i s$ operon in both E. coli and Salmonella Typhimurium. Transcription from the main promoter is repressed by Fis (negative autoregulation) and stimulated by DNA negative supercoiling. The activity of the promoter is maximal during the early stages of exponential growth and it is subject to control by the stringent response. The Fis protein influences DNA supercoiling in a number of ways. The protein is a negative regulator of the gyrA and the $\operatorname{gyr} B$ genes that encode the $\mathrm{A}$ and $\mathrm{B}$ subunits of DNA gyrase [36,37]; it is also involved in the complex regulation of the top $A$ gene that encodes the DNArelaxing topoisomerase I [38]. Fis acts as a topological buffer, constraining DNA supercoils; it can also limit access to DNA by gyrase and DNA topoisomerase I [37]. Recently, we discovered that Fis plays an important role in determining DNA supercoiling set points in Salmonella Typhimurium and E. coli [39]. E. coli displays greater sensitivity than Salmonella Typhimurium to the influences of aeration and osmotic pressure in terms of impact on its DNA supercoiling. However, if the fis gene is knocked out, this distinction is lost. The distinction can also be eliminated by the manipulation of growth conditions: when the two species are grown at high aeration, they display identical DNA topological profiles and no longer respond to osmotic pressure exerted by exposure to different concentrations of $\mathrm{NaCl}$ [39]. This shows that the degree of supercoiling of the DNA in these two bacterial species can differ or can be distinct, or can be brought into alignment by genetic methods, by manipulation of growth conditions or by a combination of the two. This illustrates the plasticity of DNA structure and its potential to influence gene expression. It suggested that a gene that has evolved to express optimally in the DNA topological milieu of Salmonella might express less well if transferred to $E$. coli, but that this might be ameliorated by manipulation of growth conditions and/or expression of the fis gene. The ssr $A$ gene encodes the histidine protein kinase that, in combination with the response regulator $\mathrm{SsrB}$, controls the transcription of the genes in SPI-2 [40]. The $s s r A$ promoter has a characteristic induction profile in Salmonella Typhimurium in which it is optimally expressed when DNA gyrase activity is impaired by drug treatment in cells growing at low osmolarity: this set of conditions is thought to mimic aspects of the intracellular 
Figure 2 DNA-binding activity of key regulatory proteins is modulated by the topology of the DNA target

The effect of DNA relaxation in vivo by inhibition of DNA gyrase activity using the antibiotic novobiocin is summarized for four Salmonella promoters and three DNA-binding proteins that bind to those promoters. The negatively supercoiled and relaxed states of the DNA are represented by the writhed and open forms of the circular DNA carrying the promoter (the angled arrow labelled ' $P$ '). The four promoters tested and their genomic locations are listed on the left and the relative binding to those promoters of the proteins Fis, H-NS and OmpR is shown in the histograms. Binding was assessed by chromatin immunoprecipitation. The data were normalized to the percentage of hilC promoter DNA precipitated by Fis. Typical data from three independent experiments are shown [18]. The results show that Fis binding is diminished, OmpR binding is enhanced, and H-NS binding is unaffected by DNA relaxation.

\section{Topological state of the DNA during ChIP-chip analysis:}

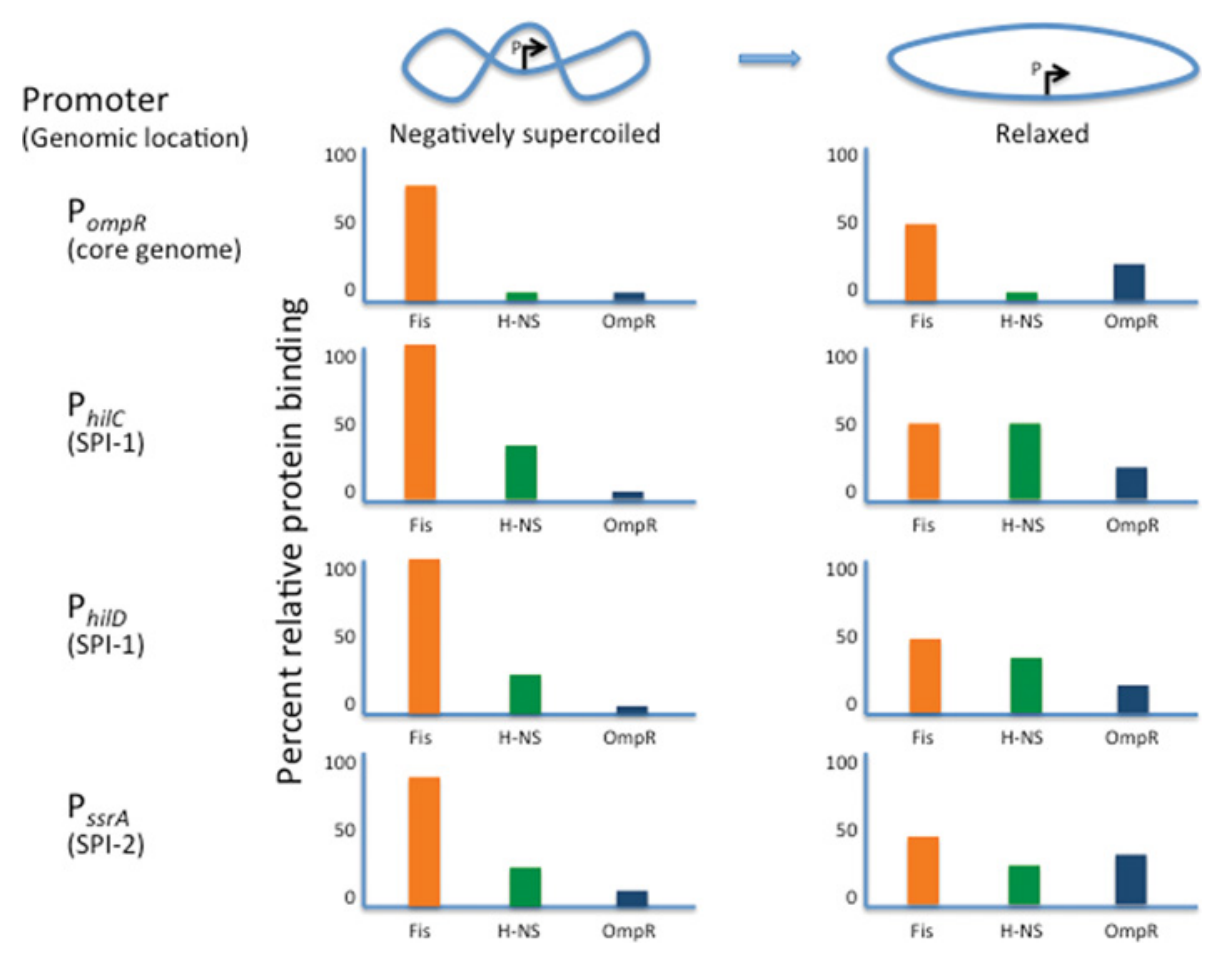

environment where $s s r A$ expression is essential for Salmonella survival [40]. The same treatment regime results in poor induction of $s s r A$ gene expression when the gene is transferred to $E$. coli. However, if the fis gene is knocked out in $E$. coli, the ssrA gene shows a response to osmotic pressure and DNA relaxation that is similar to that seen in Salmonella Typhimurium [39].

It has been shown previously that the introduction of negative supercoiling into DNA was important for the induction of transcription of the SPI-1 gene virF [41]. This hinted at the existence of a regulatory mechanism in which a transition from high to low negative supercoiling could facilitate the switch from SPI-1 to SPI-2 gene expression. Perhaps the more negatively supercoiled DNA state reflected conditions experienced in the host intestinal lumen that induced SPI-1 gene expression before invasion and the more relaxed regime that is known to characterize the DNA of Salmonella Typhimurium growing in the intravacuolar environment. We anticipated that a positive response to increased negative supercoiling would be characteristic of SPI-1 genes, whereas SPI-2 genes responded positively to DNA relaxation. This simplistic model was not supported by the experimental data [18]. Genes in both SPI-1 and SPI-2 showed a requirement for DNA relaxation as part of their induction regimes. In the case of SPI-1, this included the regulatory genes bilC and hilD [18]. This prompted us to consider the possibility that the proteins that act on DNA might respond to changes in its structure, perhaps binding more or less well depending on the superhelical density of the polymer.

\section{DNA relaxation differentially alters regulatory proteins binding}

Experiments were conducted in vivo using epitope-tagged versions of the proteins Fis, $\mathrm{H}-\mathrm{NS}$ and OmpR. In the case of $\mathrm{OmpR}$, a derivative was employed that exhibited 
constitutively the DNA-binding activity of phosphorylated OmpR. The binding of these proteins was analysed using chromatin immunoprecipitation to the promoter regions of the SPI-1 regulatory genes bilC and bilD, the SPI-2 regulatory gene $s s r A$, and the global regulatory gene $\operatorname{omp} R$ that is located in the ancestral chromosome. The supercoiling of the DNA was adjusted by inhibiting progressively the DNA supercoiling activity of DNA gyrase. The results obtained showed that the H-NS protein bound to its targets equally well regardless of the superhelicity of the DNA, the binding of the Fis protein was reduced by DNA relaxation, and the binding of the OmpR protein was enhanced by DNA relaxation [18] (Figure 2). Experiments performed in vitro with purified OmpR protein and with the DNA of the $o m p R$ promoter region supercoiled to different values of $\sigma$ also indicated a relationship between protein binding and DNA superhelical density [18].

These findings reveal that, in addition to the well-known sensitivity of DNA-based transactions (such as transcription) to changes in DNA superhelicity, there is also a marked sensitivity on the part of some DNA-binding proteins to the topological state of their DNA target. Moreover, this sensitivity varies from protein to protein, revealing a novel mechanism for the modulation of gene expression. The results described in the present paper also show that horizontally acquired genes can be subject to regulation both by proteins encoded by genes that are themselves horizontally acquired and by genes that are components of the core genome. The $\mathrm{OmpR}, \mathrm{H}-\mathrm{NS}$ and Fis proteins each have a wide tolerance for DNA sequence divergence in the DNA sites to which they bind. This presumably facilitates a relatively promiscuous relationship with DNA from all sources, leaving them well placed to influence transcription in both the core and the horizontally acquired components of the bacterial genome.

\section{Funding}

This work was supported by Science Foundation Ireland [grant number 07/IN1/B918].

\section{References}

1 De Vries, R. (2010) DNA condensation in bacteria: interplay between macromolecular crowding and nucleoid proteins. Biochimie $\mathbf{9 2}$, 1715-1721

2 Postow, L., Hardy, C.D., Arsuaga, J. and Cozzarelli, N.R. (2004) Topological domain structure of the Escherichio coli chromosome. Genes Dev. 18, 1766-1779

3 Dillon, S.C. and Dorman, C.J. (2010) Bacterial nucleoid-associated proteins, nucleoid structure and gene expression. Nat. Rev. Microbiol. 8 949-959

4 Dorman, C.J. (2009) Nucleoid-associated proteins and bacterial physiology. Adv. Appl. Microbiol. 67, 47-64

5 Hardy, C.D. and Cozzarelli, N.R. (2005) A genetic selection for supercoiling mutants of Escherichio coli reveals proteins implicated in chromosome structure. Mol. Microbiol. 57, 1636-1652

6 Luijsterberg, M.S., White, M.F., van Driel, R. and Dame, R.T. (2008) The major architects of chromatin: architectural proteins in bacteria, archaea and eukaryotes. Crit. Rev. Biochem. Mol. Biol. 43, 393-418
7 Deng, S., Stein, R.A. and Higgins, N.P. (2005) Organization of supercoil domains and their reorganization by transcription. Mol. Microbiol. 57, 1511-1521

8 Dame, R.T., Kalmykowa, 0.J. and Grainger, D.C. (2011) Chromosomal macrodomains and associated proteins: implications for DNA organization and replication in Gram-negative bacteria. PLoS Genet. 7, e1002123

9 Espéli, 0. and Boccard, F. (2006) Organization of the Escherichio coli chromosome into macrodomains and its possible functional implications. J. Struct. Biol. 156, 304-310

10 Montero Llopis, P., Jackson, A.F., Sliursarenko, O., Surovtsev, I., Heinritz, J., Emonet, T. and Jacobs-Wagner, C. (2010) Spatial organization of the flow of genetic information in bacteria. Nature $\mathbf{4 6 6}, 77-82$

11 Sobetzko, P., Travers, A. and Muskhelishvili, G. (2012) Gene order and chromosome dynamics coordinate spatiotemporal gene expression during the bacterial growth cycle. Proc. Natl. Acad. Sci. U.S.A. 109, E42-E50

12 Travers, A. and Muskhelishvili, G. (2005) Bacterial chromatin. Curr. Opin. Genet. Dev. 15, 507-514

13 Ochman, H., Lawrence, J.G. and Groisman, E.A. (2000) Lateral gene transfer and the nature of bacterial innovation. Nature 405, 299-304

14 Rhen, M. and Dorman, C.J. (2005) Hierarchical gene regulators adapt Salmonella enterica to its host milieus. Int. J. Med. Microbiol. 295 , 487-502

15 Haraga, A., Ohlson, M.B. and Miller, S.I. (2008) Salmonellae interplay with host cells. Nat. Rev. Microbiol. 6, 53-66

16 Ellermeier, J.R. and Slauch, J.M. (2007) Adaptation to the host environment: regulation of the SPI1 type III secretion system in Salmonello enterico serovar Typhimurium. Curr. Opin. Microbiol. 10, 24-29

17 Fass, E. and Groisman, E.A. (2009) Control of Salmonello pathogenicity island-2 gene expression. Curr. Opin. Microbiol. 12, 199-204

18 Cameron, A.D.S. and Dorman, C.J. (2012) A fundamental regulatory mechanism operating through OmpR and DNA topology controls gene expression in Solmonello pathogenicity islands SPI-1 and SPI-2. PLOS Genet. 8, e1002615

19 Feng, X., Oropeza, R. and Kenney, L.J. (2003) Dual regulation by phosphor-OmpR of ssrA/B gene expression in Solmonella pathogenicity island 2. Mol. Microbiol. 48, 1131-1143

20 Feng, X., Walthers, D., Oropeza, R. and Kenney, L.J. (2004) The response regulator SsrB activates transcription and binds to a region overlapping OmpR binding sites at Solmonello pathogenicity island 2. Mol. Microbiol. 54, 823-835

21 Ó Cróinín, T., Carroll, R.K., Kelly, A. and Dorman, C.J. (2006) Roles for DNA supercoiling and the Fis protein in modulating expression of virulence genes during intracellular growth of Solmonella enterica serovar Typhimurium. Mol. Microbiol. 62, 869-882

22 Schechter, L.M., Jain, S., Akbar, S. and Lee, C.A. (2003) The small nucleoid-binding proteins $\mathrm{H}-\mathrm{NS}, \mathrm{HU}$, and Fis affect hilA expression in Solmonella enterica serovar Typhimurium. Infect. Immun. 71 $5432-5435$

23 Dorman, C.J. (1991) DNA supercoiling and environmental regulation of gene expression in pathogenic bacteria. Infect. Immun. 59, 745-749

24 Dorman, C.J. and Corcoran, C.P. (2009) Bacterial DNA topology and infectious disease. Nucleic Acids Res. 37, 672-678

25 Travers, A. and Muskhelishvili, G. (2005) DNA supercoiling: a global transcriptional regulator for enterobacterial growth? Nat. Rev. Microbiol. 3, 157-169

26 Hsieh, L.S., Rouvière-Yaniv, J. and Drlica, K. (1991) Bacterial DNA supercoiling and $[$ ATP $] /[A D P]$ ratio: changes associated with salt shock. J. Bacteriol. 173, 3914-3917

27 Hsieh, L.S., Burger, R.M. and Drlica, K. (1991) Bacterial DNA supercoiling and $[A T P] /[A D P]$ : changes associated with a transition to anaerobic growth. J. Mol. Biol. 19, 443-450

28 van Workum, M., van Dooren, S.J., Oldenburg, N., Molenaar, D., Jensen, P.R., Snoep, J.L. and Westerhoff, H.V. (1996) DNA supercoiling depends on the phosphorylation potential in Escherichio coli. Mol. Microbiol. 20, 351-360

29 Dorman, C.J. (2007) H-NS, the genome sentinel. Nat. Rev. Microbiol. 5, 157-161

30 Lucchini, S., Rowley, G., Goldberg, M.D., Hurd, D., Harrison, M. and Hinton, J.C. (2006) H-NS mediates the silencing of laterally acquired genes in bacteria. PLos Pathog. 2, e81 
31 Navarre, W.W., Porwollik, S., Wang, Y, McClelland, M., Rosen, H., Libby, S.J. and Fang, F.C. (2006) Selective silencing of foreign DNA with low GC content by the H-NS protein in Solmonella. Science 313, 236-238

32 Stoebel, D.M., Free, A. and Dorman, C.J. (2008) Anti-silencing: overcoming $\mathrm{H}$-NS-mediated repression of transcription in Gram-negative bacteria. Microbiology 154, 2533-2545

33 Beach, M.B. and Osuna, R. (1998) Identification and characterization of the fis operon in enteric bacteria. J. Bacteriol. 180, 5932-5946

34 Kelly, A., Goldberg, M.D., Carroll, R.K., Danino, V., Hinton, J.C. and Dorman, C.J. (2004) A global role for Fis in the transcriptional control of metabolic and type III secretion genes of Solmonella enterica serovar Typhimurium. Microbiology 150, 2037-2053

35 Champion, K. and Higgins, N.P. (2007) Growth rate toxicity phenotypes and homeostatic supercoil control differentiate Escherichio coli from Salmonello enterica serovar Typhimurium. J. Bacteriol. 189, 5839-5849

36 Keane, 0.M. and Dorman, C.J. (2003) The gyr genes of Solmonello enterica serovar Typhimurium are repressed by the factor for inversion stimulation, Fis. Mol. Genet. Genomics 270, 369-370
37 Schneider, R., Travers, A., Kutateladze, T. and Muskhelishvili, G. (1999) A DNA architectural protein couples cellular physiology and DNA topology in Escherichio coli. Mol. Microbiol. 34, 953-964

38 Weinstein-Fisher, D. and Altuvia, S. (2007) Differential regulation of Escherichio coli topoisomerase I by Fis. Mol. Microbiol. 63, 1131-1144

39 Cameron, A.D.S., Stoebel, D.M. and Dorman, C.J. (2011) DNA supercoiling is differentially regulated by environmental factors and FIS in Escherichio coli and Solmonello enterico. Mol. Microbiol. 80, 85-101

40 Osborne, S.E. and Coombes, B.K. (2011) Transcriptional priming of solmonello pathogenicity island 2 precedes cellular invasion. PLOS ONE 6, e21648

41 Galan, J.E. and Curtiss, 3rd, R. (1990) Expression of Solmonello typhimurium genes required for invasion is regulated by changes in DNA supercoiling. Infect. Immun. 58, 1879-1885

Received 29 August 2012

doi:10.1042/BST20120222 